\title{
Metronomic chemotherapy with oral vinorelbine (mVNR) and capecitabine (mCAPE) in advanced HER2-negative breast cancer patients: is it a way to optimize disease control? Final results of the VICTOR-2 study
}

\author{
M. E. Cazzaniga ${ }^{1}$ - L. Cortesi ${ }^{2}$ - A. Ferzi $^{3}$ - L. Scaltriti ${ }^{4}$ F. Cicchiello ${ }^{1}$.

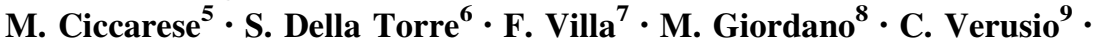 \\ M. Nicolini ${ }^{10}$ - A. R. Gambaro ${ }^{11}$ - L. Zanlorenzi ${ }^{12}$ - E. Biraghi ${ }^{13}$ - L. Legramandi ${ }^{14}$. \\ E. Rulli ${ }^{14}$ On behalf of VICTOR Study Group \\ Received: 29 September 2016/Accepted: 3 October 2016 /Published online: 17 October 2016 \\ (C) The Author(s) 2016. This article is published with open access at Springerlink.com
}

\begin{abstract}
Purpose The VICTOR-1 study demonstrated that the alloral metronomic combination of vinorelbine and capecitabine is highly active and well tolerated in hormone receptor-positive/HER2-negative patients. The VICTOR-2 study was designed to confirm these results.

Methods Patients received mVNR $40 \mathrm{mg}$ three times a week and mCAPE $500 \mathrm{mg}$ three times a day, continuously. The primary endpoint was the clinical benefit rate (CBR); secondary endpoints were toxicity, objective response rate (ORR), and progression-free survival (PFS).

Results Eighty patients were evaluable for the primary efficacy analysis. Median age was 65.3 years; most patients had HR-positive tumors $(65 \%)$. The CBR was $45.7 \%$ (95\% CI 28.8-63.4) and 51.1\% (95\% CI 35.8-66.3) in first- and $\geq$ second-line therapy, respectively. The ORR was $35.5 \%$ in first-line (95\% CI 19.2-54.6) and $25.6 \%$ in
\end{abstract}

The remaining members of the VICTOR Study Group are listed in the Appendix 1 .

M. E. Cazzaniga

marina.cazzaniga@asst-monza.it

1 Oncology Unit, ASST Monza, Via Pergolesi, 3320900 Monza, MB, Italy

2 Haematology and Oncology Unit, Azienda OspedalieroUniversitaria Policlinico di Modena, Modena, Italy

3 Oncology Unit, ASST Ovest Milanese, Ospedale di Legnano, Legnano, Italy

4 Oncology Day Hospital Unit, Ospedale Civile di Guastalla, Guastalla, Italy

5 Oncology Unit, Ospedale Vito Fazzi, Lecce, Italy

6 Oncology Unit, ASST Rhodense-Presidio di Garbagnate Milanese e Presidio di Rho, Garbagnate, Italy $\geq$ second-line (95\% CI 13.5-41.2). The median duration of response was 11.3 and 6.4 months and PFS rates at 1 year were 24.3 and $22.2 \%$, respectively. In triple-negative breast cancer patients $(N=28,35 \%)$ a lower, but clinically relevant CBR (35.7, $95 \%$ CI 18.6-55.9) was observed. The main toxicities per cycle were non-febrile neutropenia $(1.1 \%)$, hand-foot syndrome $(1.0 \%)$, nausea and vomiting $(1.0 \%)$, leucopenia $(0.8 \%)$, fatigue $(0.7 \%)$, and diarrhea $(0.4 \%)$.

Conclusion The VICTOR-2 study confirms the clinical activity of mVNR and mCAPE in HER2-negative breast cancer patients, suggesting that the easy schedule of administration, which requires monthly blood tests and limits patients' dependence on hospitals, and the low cost of the drugs are valuable elements, even for countries with limited access to innovative or expensive drugs.

Keywords Metronomic chemotherapy · Vinorelbine · Capecitabine $\cdot$ Breast cancer

\footnotetext{
Oncology Unit, ASST, Lecco, Italy

8 Oncology Unit, ASST Lariana, Como, Italy

9 Oncology Unit, ASST della Valle Olona, Saronno, Italy

10 Oncology Day Hospital Unit, Azienda USL Romagna, Cattolica, Italy

11 Oncology Unit, ASST Fatebenefratelli-Sacco, Milan, Italy

12 Oncology Unit, ASST della Valle Olona, Busto Arsizio, Italy

13 Oncology Unit, ASST Melegnano e Martesana, Gorgonzola, Italy

14 Methodology for Clinical Research Laboratory, IRCCS Istituto di Ricerche Farmacologiche Mario Negri, Milan, Italy
} 


\section{Introduction}

One of the emerging strategies to achieve disease control in advanced breast cancer while reducing the impact of toxicity is metronomic chemotherapy (mCT) [1]. mCT refers to the optimal biological dose, defined as the minimum biologically effective dose of a chemotherapeutic agent given as a continuous dosing regimen, with no prolonged drug-free breaks, that leads to anti-tumor activity [2].

A strong rationale supports the choice of a combination regimen when using a metronomic schedule: data from preclinical studies suggest that the metronomic combination of two different drugs allows the use of lower doses while still having an anti-tumor effect [3].

Several phase II studies have investigated metronomic vinorelbine $(\mathrm{mVNR})$ in the treatment of breast cancer [4-8]. mVNR demonstrated long-lasting disease control combined with a good toxicity profile. Furthermore, a synergistic effect has been shown for VNR and capecitabine (CAPE), even when administered at standard schedules and doses $[9,10]$.

Our group recently published the results of the VICTOR-1 study, showing that the all-oral metronomic combination of VNR and CAPE is highly active in a population of hormone receptor (HR)-positive/HER2-negative advanced breast cancer patients, with a very low incidence of Grade 3-4 toxicity [4].

The phase II VICTOR-2 study was designed with the aim of confirming the results of the previous trial in a larger cohort of breast cancer patients.

\section{Patients and methods}

VICTOR-2 is an open-label, phase II, multicenter trial conducted in 12 Italian centers between August 2011 and May 2015.

The study was conducted in accordance with the 1987 Declaration of Helsinki and adhered to Good Clinical Practice guidelines. Approval of the protocol was obtained from the local ethics committee for each participating center; all patients were required to give written informed consent before enrolment and to comply with the protocol for the duration of the study.

\section{Patients}

Eligible patients were female, $\geq 18$ years, with documented locally advanced, metastatic breast cancer, both previously treated or chemotherapy-naïve. Other inclusion criteria included HER2-negative disease (IHC $0-1$ or IHC 2, confirmed as FISH negative), $\geq 1$ measurable lesion according to RECIST 1.0 criteria and a life expectancy of $\geq 16$ weeks. Previous endocrine therapy for advanced disease was allowed. Patients were required to have adequate bone marrow, hepatic, and renal functions, indicated by hemoglobin $\geq 10 \mathrm{~g} \times 100 \mathrm{~mL}$, absolute neutrophil count $\geq 2 \times 10^{9} / \mathrm{L}$, platelet count $\geq 100 \times 10^{9} / \mathrm{L}$, total serum bilirubin $<1.5 \times$ upper normal limit (UNL), AST/ALT $<2.5 \times \mathrm{UNL},(<3.5 \times \mathrm{UNL}$ for liver metastases $)$, and alkaline phosphatase $<2.5 \times$ UNL $(<5 \times$ UNL for bone metastases).

Patients were ineligible if they had only local relapse, previous exposure to a vinca alkaloid or CAPE, serious comorbidities such as cardiac disease, uncontrolled diabetes or hypercalcemia, severe peripheral neuropathy, active infection, or previous organ allograft. Patients were also excluded if they were pregnant or lactating; had clinical central nervous system or leptomeningeal metastases, a malabsorption disease, hypersensitivity to fluoropyrimidine therapy; had participated in another clinical trial with any investigational drug within 30 days before study inclusion; or had a history of another malignancy. Drugs acting on P450 cytochrome were not allowed during the study.

Patients were divided in two groups, according to treatment line $\quad($ first-line $=$ Group $1 ; \geq$ second-line $=$ Group 2).

\section{Treatment and dose modifications}

Treatment consisted of VNR $40 \mathrm{mg}$ each alternative day of the week (Monday, Wednesday, and Friday) and CAPE $500 \mathrm{mg}$ three times a day (TID) after meals, given continuously without drug-free periods, until disease progression, unacceptable toxicity, or patient's refusal. Patients' compliance was evaluated by a diary given at the beginning of each cycle ( 1 cycle $=3$ weeks). The dose of VNR was temporarily reduced to $30 \mathrm{mg}$ three times a week at the first appearance of Grade 2 neutropenia or thrombocytopenia; the dose was increased to the previous level $(40 \mathrm{mg}$ ) only if a complete recovery was observed at the beginning of the subsequent cycle. If a second episode of Grade 2 neutropenia or thrombocytopenia occurred, the dose was maintained at $30 \mathrm{mg}$ until the end of the study with no further reduction. In the case of Grade 3-4 neutropenia or thrombocytopenia, VNR was interrupted for a maximum of 3 weeks, until recovery of neutrophil count at $1.0 \times 10^{9} / \mathrm{L}$; the dose administered upon resuming treatment was determined according to the toxicity grade. CAPE was reduced to $1000 \mathrm{mg} /$ day in case of Grade 3-4 neutropenia or thrombocytopenia, or Grade 2-3 diarrhea or hand-foot syndrome, until recovery to Grade 1. For any other Grade 3-4 toxicity, both drugs were interrupted until recovery to lower grade. 


\section{Outcomes}

The primary endpoint of the study was CBR, defined as the proportion of patients with complete (CR) or partial response $(\mathrm{PR})$ or with stable disease $(\mathrm{SD})$ at 24 weeks from the start of treatment. Patients without a computed tomography (CT) re-evaluation at week 24 were considered non-responder if they discontinued treatment for medical decision, clinical progression, death, or toxicity.

Secondary endpoints were the objective response rate (ORR) and disease control rate (DCR), defined as the percentage of patients with $\mathrm{CR}+\mathrm{PR}$ or $\mathrm{CR}+\mathrm{PR}+\mathrm{SD}$, respectively, according to RECIST criteria. Further assessments included disease-free interval (DFI), progression-free survival (PFS), and time to progression (TTP). For patients achieving a CR or PR, the time to response and duration of response were also assessed.

\section{Assessments}

Blood tests evaluating hepatic and renal function together with CEA and CA 15.3 were conducted at baseline and every 3 cycles, until study end. For each cycle white blood cells, erythrocyte count, hemoglobin, and platelets were assessed, before chemotherapy delivery. Tumor status was assessed according to RECIST 1.0 criteria, every 3 cycles (9 weeks) until disease progression, interruption of the treatment for toxicity, or patient's refusal.

\section{Statistical methods}

A sample size for each group has been defined according to the Fleming approach, modified by A'Hern [11]. We assumed the treatment had no therapeutic interest with a $\mathrm{CBR} \leq 40 \%$ for Group 1 and $\leq 20 \%$ for Group 2, while a $\mathrm{CBR} \geq 55 \%$ and $\geq 35 \%$, respectively, was required to consider the treatment active. With a one-sided alpha level of $10 \%$ and a power of $85 \%$, a total of 61 patients in Group 1 and 49 patients in Group 2 had to be enrolled. Considering a possible drop-out of about $10 \%, 120$ patients were required to have 105 patients evaluable for the primary endpoint (60 in Group 1 and 45 in Group 2).

CBR, ORR, and DCR were given as point estimate and $95 \%$ confidence interval $(\mathrm{CI})$. CIs were computed using exact binomial methods. Subjects who were not reported as having died or with progression/relapse at the time of the analysis were censored at their last available contact date. Survival data were summarized by median and interquartile range (IQR), computed with the Kaplan-Meier method. A Cox proportional hazard model was used to assess the impact of clinical factors on survival endpoints and results were expressed as hazard ratios (HR) and $95 \%$ CI. Compliance with treatment and toxicity were evaluated using both cycle and patient as units of analysis. All analyses were conducted on the whole population and according to treatment line. Exploratory analyses were conducted on subgroups defined by HR status and metastatic site. Analyses were carried out with SAS (Version 9.2).

\section{Results}

\section{Patient characteristics}

Between August 2011 and May 2015, 86 patients were enrolled. Six patients were subsequently excluded from the analysis, due to screening failure $(N=4)$ and missing data $(N=2)$ (Fig. 1). After the enrollment of 35 patients in Group 1 and 45 in Group 2, the study was prematurely closed, due to the slow recruitment of first-line patients. The minimum number of responding patients required to demonstrate clinical activity was reached in Group 2; thus enrollment was simultaneously closed in both groups. With 35 patients enrolled in Group 1, the power to test the original hypothesis is about $65 \%$; the purpose of analysis in this group, therefore, is merely descriptive.

The median age was 65.3 (56.0-69.3) years; most patients $(65 \%)$ had HR-positive disease (Table 1). Median DFI was 4.3 years (IQR 2.1-10.6). Sixty patients (76.9\%) had $>2$ metastatic sites and $58(72.6 \%)$ had visceral disease. At enrollment, 70 patients $(87.5 \%)$ had already received at least one prior antiblastic regimen; 35 patients $(43.7 \%)$ had been treated with anthracyclines, taxanes, or the combination.

\section{Treatments received}

A total of 868 cycles were administered with a median of 9 (range 1-59) cycles per patient. A full dose of both drugs was administered for $76.5 \%$ of cycles (89.2 and $67.8 \%$ in Group 1 and 2, respectively). Forty-two patients $(52.6 \%$ ) had dose reduction of the study drugs. At the final analysis, treatment had been discontinued in 75 patients for disease progression or death $(N=61,81.4 \%)$, toxicity $(N=8$, $10.7 \%)$, or physician decision $(N=5,6.7 \%)$.

\section{Efficacy}

The CBR was $48.8 \%$ (95\% CI 37.4-60.2) in the overall population, $45.7 \%$ (95\% CI 28.8-63.4) in Group 1, and $51.1 \%$ (95\% CI 35.8-66.3) in Group 2 (Table 2). Regarding receptor status, the CBR was $55.8 \%(95 \% \mathrm{CI}$ 41.3-69.5) in HR-positive patients and 35.7\% (95\% CI 18.6-55.9) in triple-negative breast cancer (TNBC) patients; according to metastatic site the CBR was $59.1 \%(95 \%$ CI 36.4-79.3) in patients without visceral involvement and 


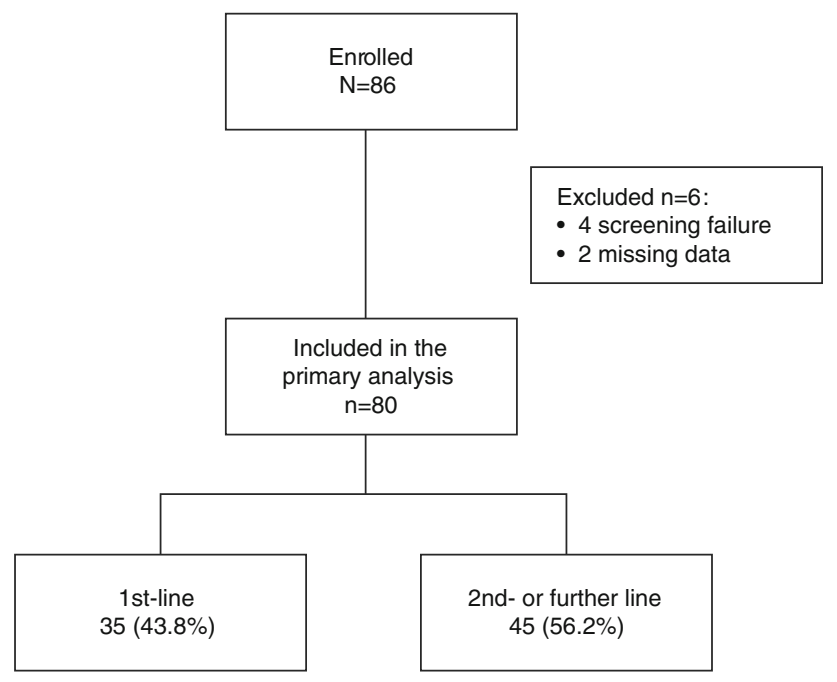

$44.8 \%$ (95\% CI 31.7-58.5) in those with such involvement. The median duration of $\mathrm{CB}$ was 5.8 months (IQR 2.3-14.3) in Group 1 and 6.0 months (IQR 3.7-15.5) in Group 2.

Patients in Group 1 had higher ORR (35.5\%, $95 \%$ CI 19.2-54.6) and DCR (74.2, $95 \%$ CI 55.4-88.1) than those in Group 2 (ORR 25.6, $95 \%$ CI 13.5-41.2, DCR 67.4, $95 \%$ CI 51.5-80.9) (Table 3). The median time to response was comparable in the two groups (2.1 months overall; IQR 2.1-4.1). The median duration of response was 11.3 and 6.4 months for Group 1 and Group 2, respectively.

The median TTP was 7.9 months (IQR 5.3-12.8) in Group 1 and 7.2 months (IQR 2.8-11.5) in Group 2. No difference in TTP was observed according to metastatic site or biological subtype (Table 4).

Fig. 1 Study population

Table 1 Patients and tumor characteristics

\begin{tabular}{|c|c|c|c|}
\hline & First-line $N=35$ & Second-line $N=45$ & Overall $N=80$ \\
\hline Number of patients $N(\%)$ & $35(43.8)$ & $45(56.2)$ & 80.0 \\
\hline \multicolumn{4}{|l|}{ Age (years) } \\
\hline Median (Q1-Q3) & $66.3(56.4-76.5)$ & $64.9(55.7-68.2)$ & $65.3(56.0-69.3)$ \\
\hline Min-Max & $38.0-85.6$ & $44.0-82.7$ & $38.0-85.6$ \\
\hline \multicolumn{4}{|l|}{ Receptor status $(N) \%$} \\
\hline HR-positive & $22(62.9)$ & $30(66.7)$ & $52(65.0)$ \\
\hline Triple-negative & $13(37.1)$ & $15(33.3)$ & $28(35.0)$ \\
\hline \multicolumn{4}{|l|}{ Metastatic site $N(\%)$} \\
\hline Only bone with/without other site & $9(25.7)$ & $8(17.8)$ & $17(21.2)$ \\
\hline Only visceral with/without other site & $13(37.1)$ & $16(35.6)$ & $29(36.3)$ \\
\hline Visceral and bone with/without other site & $10(28.6)$ & $19(42.2)$ & $29(36.3)$ \\
\hline Other site & $3(8.6)$ & $2(4.4)$ & $5(6.2)$ \\
\hline \multicolumn{4}{|l|}{ Number of metastatic sites $N(\%)$} \\
\hline 1 & $2(6.1)$ & $3(6.7)$ & $5(6.4)$ \\
\hline 2 & $3(9.1)$ & $10(22.2)$ & $13(16.7)$ \\
\hline$>2$ & $28(84.8)$ & $32(71.1)$ & $60(76.9)$ \\
\hline Not reported & 2 & 0 & 2 \\
\hline \multicolumn{4}{|l|}{ Chemotherapy for metastatic tumor* $N(\%)$} \\
\hline Yes & $0(0.0)$ & $38(86.4)$ & $38(54.3)$ \\
\hline No & $26(100)$ & $6(13.6)$ & $32(45.7)$ \\
\hline \multicolumn{4}{|l|}{ Metastatic treatment $N(\%)$} \\
\hline Only anthracyclines & & $1(2.6)$ & $1(2.6)$ \\
\hline Only taxanes & & $7(18.4)$ & $7(18.4)$ \\
\hline Only other & & $3(7.9)$ & $3(7.9)$ \\
\hline Anthracyclines and taxanes & & $8(21.1)$ & $8(21.1)$ \\
\hline Anthracyclines and other & & $2(5.3)$ & $2(5.3)$ \\
\hline Taxanes and other & & $12(31.6)$ & $12(31.6)$ \\
\hline Anthracyclines and taxanes and other & & $5(13.2)$ & $5(13.2)$ \\
\hline
\end{tabular}

$N$ total number of subjects, $Q 1-Q 3$ first-third quartile, Min-Max minimum-maximum value

* Among the 70 patients that had already received at least one prior antiblastic regimen at study enrollment (either in the adjuvant and/or the metastatic setting) 
After a median follow-up of 18 months, 65 patients progressed, and 6 died. The median PFS was 6.7 months (IQR 4.74-11.3) in Group 1 and 7.2 months (95\% CI 2.8-11.5) in Group 2 while, according to biological type, the median PFS was 8.2 months in HR-positive patients and 4.7 months in TNBC patients (Fig. 2). PFS rate at 1 year according to the line of treatment was 24.3 and $22.2 \%$ for Group 1 and 2, respectively.

\section{Treatment-related toxicities}

The most frequent severe (Grade 3-5) toxicities in the 896 cycles delivered were non-febrile neutropenia $(1.1 \%$ of cycles), hand-foot syndrome (1.0\%), nausea/vomiting $(1.0 \%)$, leucopenia $(0.8 \%)$, fatigue $(0.7 \%)$, and diarrhea $(0.4 \%)$ (Table 5). No severe alopecia was observed.

Severe hematologic toxicities per patient included Grade 3-4 leucopenia $(N=7$ patients, $8.8 \%)$, febrile neutropenia $(N=4,5 \%)$, Grade 3-4 thrombocytopenia $(N=2,2.5 \%$; however, no bleeding occurred), and Grade 3 anemia $(N=1,1.3 \%)$. Among severe non-hematological toxicities, nausea/vomiting (10.0\%), hand-foot syndrome $(10.0 \%)$, fatigue $(6.3 \%)$, and diarrhea $(5.0 \%)$ were the most common.

Most of Grade 3-4 events occurred during the first 3 cycles, after which the probability of an adverse event per treatment cycle dropped to $<1 \%$.

\section{Discussion}

To our knowledge, the VICTOR-2 study is the first multicenter prospective trial testing the fully oral metronomic combination of VNR and CAPE in a population of advanced HER2-negative breast cancer patients with prespecified analyses of efficacy and safety according to

Table 2 Clinical benefit rate according to pre-specified subgroups

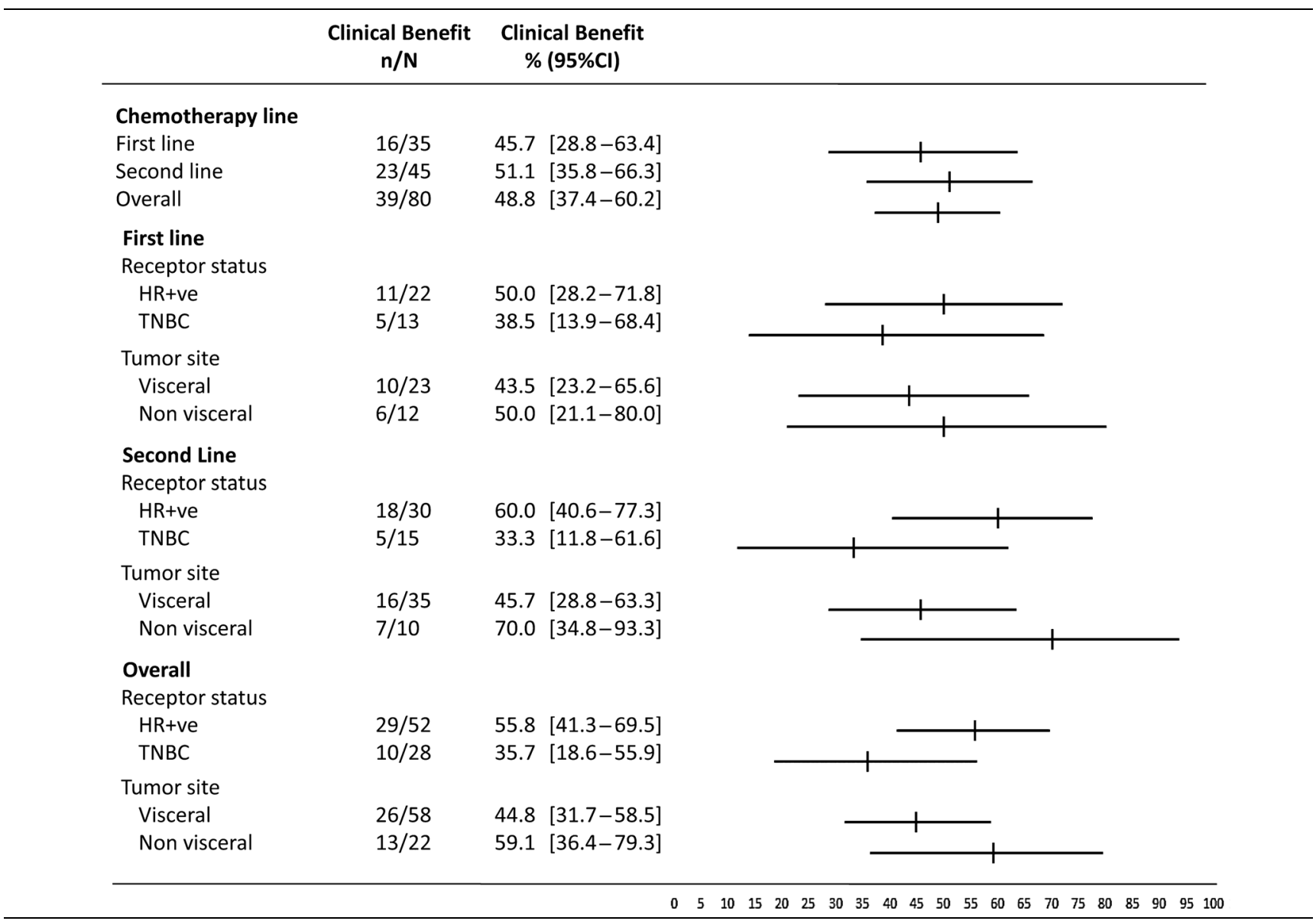

$N$ total number of subjects, $95 \%$ CI $95 \%$ confidence interval, $H R$ hormone receptor, TNBC triple-negative breast cancer 
Table 3 Objective response rate, disease control rate, duration of disease control, and time to response

\begin{tabular}{|c|c|c|c|}
\hline Objective response rate (ORR) & $\begin{array}{l}\text { First-line (Group 1) } \\
N=31\end{array}$ & $\begin{array}{l}\text { Second-line (Group 2) } \\
N=43\end{array}$ & $\begin{array}{l}\text { Overall } \\
N=74\end{array}$ \\
\hline Responders (CR + PR): $n(\%)$ & $11(35.5)$ & $11(25.6)$ & $22(29.7)$ \\
\hline$[95 \% \mathrm{CI}]$ & {$[19.2-54.6]$} & {$[13.5-41.2]$} & {$[19.7-41.5]$} \\
\hline \multicolumn{4}{|l|}{ Disease control rate (DCR) } \\
\hline Responders $(\mathrm{CR}+\mathrm{PR}+\mathrm{SD}): n(\%)$ & $23(74.2)$ & $29(67.4)$ & $52(70.3)$ \\
\hline \multirow[t]{2}{*}[95\%\mathrm{CI}]{} & {$[55.4-88.1]$} & {$[51.5-80.9]$} & {$[58.5-80.3]$} \\
\hline & $\begin{array}{l}\text { First-line (Group 1) } \\
N=11\end{array}$ & $\begin{array}{l}\text { Second-line (Group 2) } \\
N=11\end{array}$ & $\begin{array}{l}\text { Overall } \\
N=22\end{array}$ \\
\hline \multicolumn{4}{|l|}{$\begin{array}{l}\text { Kaplan-Meier estimate of duration } \\
\text { of objective response (months) }\end{array}$} \\
\hline Median & 11.3 & 6.4 & 8.2 \\
\hline (IQR) & 4.1-not reached & $5.3-12.8$ & $5.2-12.8$ \\
\hline \multicolumn{4}{|l|}{ Time to objective response (months) } \\
\hline Median & 2.1 & 2.1 & 2.1 \\
\hline (IQR) & $2.1-5.0$ & $2.1-3.4$ & $2.1-4.1$ \\
\hline
\end{tabular}

$N$ total number of subjects, $I Q R$ interquartile range, $95 \% C I 95 \%$ confidence interval, $C R$ complete response, $P R$ partial response, $S D$ stable disease

Table 4 Time to progression (TTP) in the whole population and according to hormone receptor status

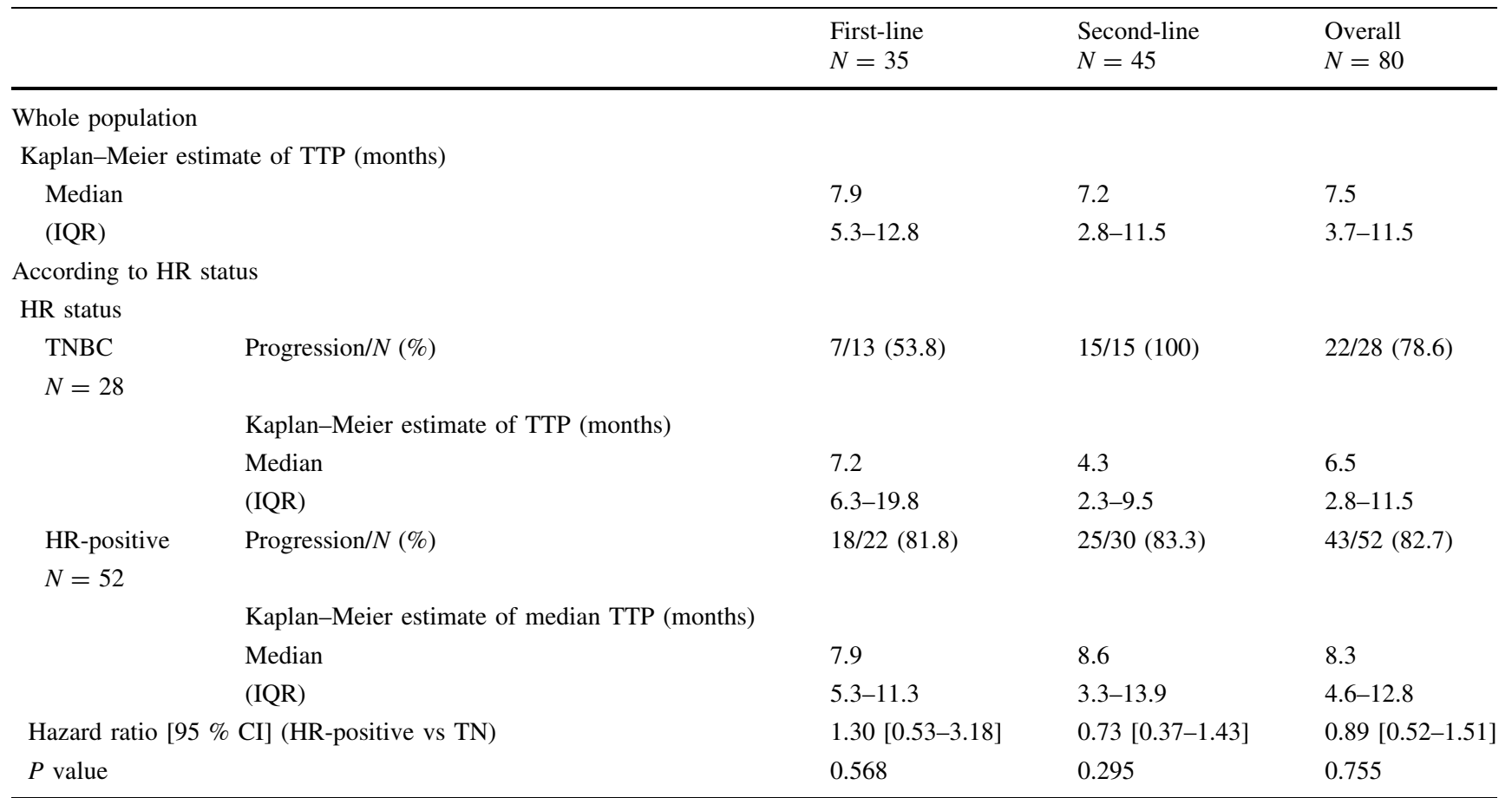

$N$ total number of subjects, IQR Interquartile range, $H R$ hormone receptor, TNBC triple-negative breast cancer, TTP time to progression, $95 \%$ CI $95 \%$ confidence interval

biological subtype, line of treatment, and site of metastatic disease.

The metronomic combination of continuous oral VNR and CAPE resulted in a promising CBR in pretreated patients $(51.1 \%)$, while results for first-line patients, due to premature recruitment closure, are inconclusive. ORR and particularly DCR are of relevant clinical interest due to the low incidence of serious adverse events.

Several phase II studies have tested the metronomic administration of oral anticancer drugs, reporting CBRs of 31-53\% and ORRs of 19-52\% [12-14]. Most of these studies had small sample sizes and were conducted in 
Fig. 2 Progression-free survival according to treatment line and hormone receptor status

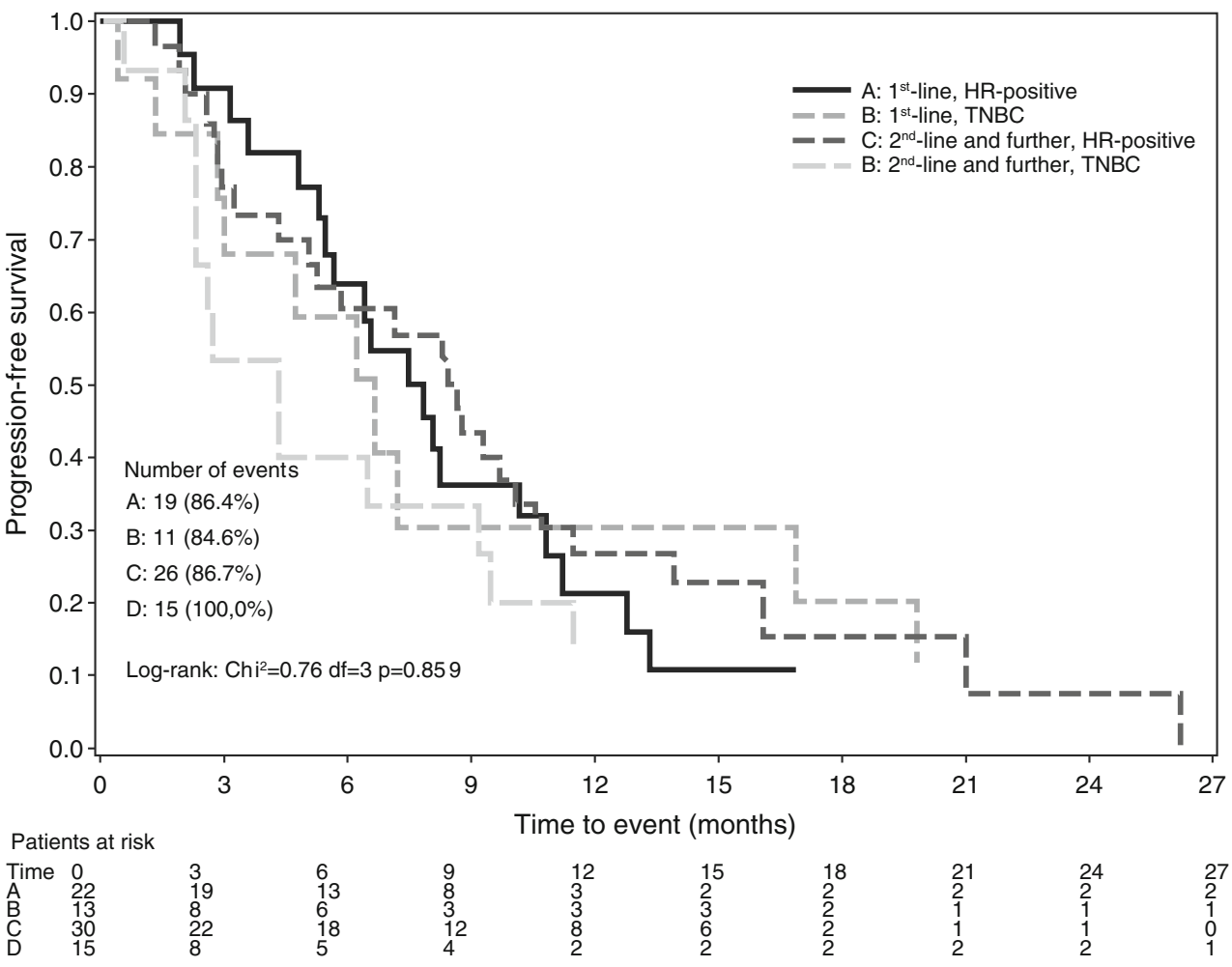

Legend: HR: hormone receptor; TNBC: triple-negative breast cancer
Table 5 Percentages of G3 + G4 + G5 toxicity in treatment cycles. (Unit of analysis $=$ cycle)

\begin{tabular}{llll}
\hline & First-line & Second-line & Overall \\
& $N=365$ & $N=531$ & $N=896$ \\
\hline Non-febrile neutropenia & 1.6 & 0.8 & 1.1 \\
Hand and foot syndrome & 0.5 & 1.3 & 1.0 \\
Nausea and vomiting & 0.8 & 1.2 & 1.0 \\
Leucopenia & 1.1 & 0.6 & 0.8 \\
Fatigue & 1.1 & 0.4 & 0.7 \\
Diarrhea & 0.5 & 0.4 & 0.4 \\
Febrile neutropenia & 0.5 & 0.4 & 0.4 \\
Allergic reaction & 0.8 & 0.0 & 0.3 \\
Mucositis & 0.5 & 0.0 & 0.2 \\
Thrombocytopenia & 0.0 & 0.4 & 0.2 \\
Anemia & 0.3 & 0.0 & 0.1 \\
Fever/infection & 0.0 & 0.0 & 0.0 \\
Alopecia & 0.0 & 0.0 & 0.0 \\
Total & 7.7 & 5.5 & 6.4 \\
\hline
\end{tabular}

$N$ total number of cycles

heavily pretreated breast cancer patients; in some cases the schedule could not be defined as metronomic, making comparison difficult.

More recent trials tested different and more active drugs, mainly VNR and CAPE, reporting CBRs of $77-80 \%$ and
ORRs of approximately $50 \%$. In a small, single-center study of 34 elderly patients with metastatic breast cancer treated with oral mVNR, ORR was $38 \%$ and CBR was $68 \%$ [15]. Most patients were receiving the treatment in the firstline setting and this may partially account for the high CBR. In another study [6], the combination of a low protracted dose of temozolomide, mVNR, and radiotherapy for newly diagnosed brain metastases from breast cancer resulted in CBR of $77 \%$ and ORR of $52 \%$. Finally, the single-center phase I/II VICTOR-1 study [4] reported similar results to those shown by other studies with a clinical benefit rate (CBR) of $58.1 \%$. Taken together, these data and those from the present study suggest that $\mathrm{mCT}$, when administered with highly active and synergistic drugs such as VNR and CAPE, is able to induce encouraging DCR.

The VICTOR-2 trial is the first study reporting data on the activity of metronomic VNR and CAPE in TNBC patients, a population for which there is a strong medical need for safe and active treatments. CBR in this population was $35.7 \%$ and median duration of CB was 11.3 months. The median time to objective response was 2.1 months: this finding is of particular importance in the presence of aggressive disease, as it suggests that $\mathrm{mCT}$ could be an option even in this subset of patients, debunking the myth that it should be reserved for heavily pretreated patients, for whom no other therapeutic options are available. 
The incidence of Grade 3-4 toxicity was very low with the metronomic combination of VNR and CAPE $(6.4 \%$ in 896 cycles). These results are in accordance with those from the other studies [4, 5] and are particularly important considering that half of the patients were pretreated with at least one line of CT and the majority $(72.6 \%)$ had visceral involvement.

The results of this study indicate that there are no drugcumulative effects: the highest incidence of serious events was observed in cycles 1-3 and 4-6 and was followed by a significant decrease in severe toxicity during the subsequent cycles. The lack of drug accumulation over time, at least for VNR, has previously been demonstrated [16]. The availability of active and highly tolerated metronomic regimens, such as the VICTOR combination, may allow long-term therapy.

In this study, DCR was $74.2 \%$ in Group 1 and $67.4 \%$ in Group 2 and median duration of disease control was 7.6 months; these results suggest the metronomic VICTOR combination represents a feasible option to optimize the balance between efficacy and tolerability. Furthermore, the long-lasting treatment with mVNR and mCAPE could account for the high PFS reported in our study, with more than a quarter of the patients alive and free from progression after 12 months of mCT.

The ever-increasing published evidence on the use of mCT [17], may now contribute to outlining the profile of the patients who are likely to benefit from this option: HRpositive tumors, indolent disease, and bone metastases are all characteristics well represented in the metronomic studies and should be considered for patient's selection. In addition, the results from this study support the use of the metronomic VICTOR combination in first-line.

This fully oral therapy does not require frequent blood testing, and the easy schedule of administration means that patients can remain at home for the whole duration of treatment. Positive clinical outcomes as first-line therapy, together with very low toxicity, mean that metronomic regimens could serve as a bridge to transition HR-positive patients from endocrine therapy to more aggressive CT regimens. Furthermore, lack of alopecia, severe nausea, and vomiting and the very low incidence of severe complications are an added value in the palliative setting.

\section{Conclusion}

The results of the VICTOR-2 study have demonstrated the efficacy and safety of the metronomic combination of VNR and CAPE in an unselected group of patients with metastatic breast cancer, strongly suggesting that continuous administration of low-dose drugs allows prolonged duration of treatment and positive clinical outcomes, while minimizing the risk of adverse events.

Acknowledgments The authors would like to thank Monica Perez Gila for Data Management support. They also thank Cécile Duchesnes of Springer Healthcare Communications, who provided technical and English editing of the manuscript; this medical writing assistance was funded by Pierre-Fabre, Italy.

Funding This work was supported by a grant from Associazione Onlus "Don Giulio Farina per la Cura e la Ricerca dei Tumori," Monza, Italy. None of the authors has a financial relationship with this organization.

\section{Compliance with Ethical Standards}

Conflict of interest The authors have declared no conflicts of interest.

Informed consent The study was conducted in accordance with the 1987 Declaration of Helsinki and adhered to Good Clinical Practice guidelines. Approval of the protocol was obtained from the local ethics committee for each participating center; all patients were required to give written informed consent before enrolment and to comply with the protocol for the duration of the study.

Open Access This article is distributed under the terms of the Creative Commons Attribution 4.0 International License (http://crea tivecommons.org/licenses/by/4.0/), which permits unrestricted use, distribution, and reproduction in any medium, provided you give appropriate credit to the original author(s) and the source, provide a link to the Creative Commons license, and indicate if changes were made.

\section{Appendix}

List of co-authors belonging to the VICTOR Study Group Francesca Riva, Monza, Italy; Davide Pelizzoni, Monza, Italy; Isabella Marchi, Modena, Italy; Elena Collovà, Legnano, Italy; Giuseppe Prati, Guastalla, Italy; Antonio Ardizzoia, Lecco, Italy; Davide Toniolo, Rho, Italy; Palma Pugliese, Como, Italy; Claudia Pogliani, Saronno, Italy; Abbondanza Gambino, Lecce, Italy; Lucia Stocchi, Cattolica, Italy; Andrea Colombo, Busto Arsizio, Italy; Cinzia Fasola, Milano, Italy; Raffaele Venezia, Gorgonzola, Italy; Fabio Galli, Milano, Italy; Valter Torri, Milano, Italy.

\section{References}

1. Cazzaniga ME, Camerini A, Addeo R et al (2016) Metronomic oral vinorelbine in advanced breast cancer and non-small-cell lung cancer: current status and future development. Futur Oncol 12:373-378

2. Di Desidero T, Xu P, Man S et al (2015) Potent efficacy of metronomic topotecan and pazopanib combination therapy in 
preclinical models of primary or late stage metastatic triplenegative breast cancer. Oncotarget 6:42396-42410

3. Kareva I, Waxman DJ, Lakka Klement G (2015) Metronomic chemotherapy: an attractive alternative to maximum tolerated dose therapy that can activate anti-tumor immunity and minimize therapeutic resistance. Cancer Lett 358:100-106

4. Cazzaniga ME, Torri V, Villa F et al (2014) Efficacy and safety of the all-oral schedule of metronomic vinorelbine and capecitabine in locally advanced or metastatic breast cancer patients: the phase I-II VICTOR-1 study. Int J Breast Cancer 2014:769790

5. Montagna E, Lai A, Palazzo A et al (2015) A phase II study of metronomic oral chemotherapy for metastatic breast cancer patients: Safety and efficacy results of vinorelbine, cyclophosphamide plus capecitabine (VEX) combination. Eur J Cancer Congr 51:S291-S292

6. Addeo R, Sperlongano P, Montella L et al (2012) Protracted low dose of oral vinorelbine and temozolomide with whole-brain radiotherapy in the treatment for breast cancer patients with brain metastases. Cancer Chemother Pharmacol 70:603-609

7. Saloustros E, Kalbakis K, Vardakis N et al (2011) Metronomic vinorelbine plus bevacizumab as salvage therapy for patients with metastatic breast cancer. J BUON 16:215-218

8. De Iuliis F, Salerno G, Taglieri L et al (2015) On and off metronomic oral vinorelbine in elderly women with advanced breast cancer. Tumori J 101:30-35

9. Tubiana-Mathieu N, Bougnoux P et al (2009) All-oral combination of oral vinorelbine and capecitabine as first-line chemotherapy in HER2-negative metastatic breast cancer: an International Phase II trial. Br J Cancer 101:232-237
10. Nolè F, Crivellari D, Mattioli R et al (2009) Phase II study of an all-oral combination of vinorelbine with capecitabine in patients with metastatic breast cancer. Cancer Chemother Pharmacol 64:673-680

11. A'Hern RP (2001) Sample size tables for exact single-stage phase II designs. Stat Med 20:859-866

12. Colleoni M, Rocca A, Sandri MT et al (2002) Low-dose oral methotrexate and cyclophosphamide in metastatic breast cancer: antitumor activity and correlation with vascular endothelial growth factor levels. Ann Oncol 13:73-80

13. Orlando L, Cardillo A, Ghisini R et al (2006) Trastuzumab in combination with metronomic cyclophosphamide and methotrexate in patients with HER-2 positive metastatic breast cancer. BMC Cancer 6:225

14. Wang Z, Lu J, Leaw S et al (2012) An all-oral combination of metronomic cyclophosphamide plus capecitabine in patients with anthracycline- and taxane-pretreated metastatic breast cancer: a phase II study. Cancer Chemother Pharmacol 69:515-522

15. Addeo R, Sgambato A, Cennamo G et al (2010) Low-dose metronomic oral administration of vinorelbine in the first-line treatment of elderly patients with metastatic breast cancer. Clin Breast Cancer 10:301-306

16. Gennari A, Stockler M, Puntoni M et al (2011) Duration of chemotherapy for metastatic breast cancer: a systematic review and meta-analysis of randomized clinical trials. J Clin Oncol 29:2144-2149

17. Lien K, Georgsdottir S, Sivanathan L et al (2013) Low-dose metronomic chemotherapy: a systematic literature analysis. Eur J Cancer 49:3387-3395 